\title{
Allgemeiner Teil Sozialversicherung: weniger Inkassoprobleme im Tiers garant
}

Robert Gmür, Rechtsdienst FMH

Am 1. Januar 2003 ist das Bundesgesetz über den allgemeinen Teil des Sozialversicherungsrechts (ATSG) in Kraft getreten. Gleichzeitig hat der Bundesrat die dazugehörige Verordnung (ATSV) erlassen und zahlreiche Ausführungsbestimmungen in der KVV angepasst.

\section{Überblick}

Das Wichtigste vorweg: Das sogenannte Arztrecht, also das Tarifvertragsrecht und die Bestimmungen über die Zulassung von Ärzten zur Sozialversicherung, wird vom ATSG vollständig ausgeklammert. In diesem für die Ärzteschaft zentralen Punkt ändert sich mit andern Worten nichts.

Nachdem sich das Parlament während 15 Jahren mit dem ATSG beschäftigt und mehr oder weniger schwergetan hat, wurde eine gegenüber dem ursprünglichen Entwurf weniger ehrgeizige «Light-Version» verabschiedet. Betroffen sind alle Sozialversicherungszweige mit Ausnahme der beruflichen Vorsorge. Die wesentlichen Neuerungen sind:

- Die Versicherten haben gegenüber allen Sozialversicherern ein Recht auf Aufklärung und Beratung; bisher war das explizit nur in der Krankenversicherung vorgesehen.

- Neu gilt ein einheitliches Verwaltungsverfahren. Hervorzuheben ist, dass Versicherte nun in allen Sozialversicherungszweigen gegen Verfügungen Einsprache erheben können. Das war bisher nur in der Kranken- und Unfallversicherung vorgesehen. Einsprache heisst, dass die Versicherten ihren Standpunkt zunächst nochmals gegenüber dem Versicherer verdeutlichen können, ohne gleich ein Gerichtsverfahren anhängig machen zu müssen; auf der anderen Seite kann der zusätzliche Verfahrensschritt eine Verlängerung der beispielsweise in der IV schon problematisch langen Verfahren mit sich bringen. Man wird die praktischen Erfahrungen abwarten müssen, um die konkreten Auswirkungen beurteilen zu können.
- Leistungsstreitigkeiten zwischen Versicherern und Versicherten können neu mit einem Vergleich beigelegt werden. Allerdings dürfen auch im Rahmen eines Vergleichs keine gesetzlich unzulässigen Leistungen vereinbart werden. Das BSV hat angekündigt, entsprechende Leitlinien zu erarbeiten.

- Das ATSG bringt eine verbesserte Koordination der Ansprüche unter Sozialversicherungen und gegenüber haftpflichtigen Dritten. Neu gilt überall das sogenannte Überentschädigungsverbot, zudem ein übergreifendes Vorleistungssystem, das bestimmt, welche Sozialversicherung im Zweifelsfall vorleistungspflichtig ist.

- Schliesslich führt das ATSG eine (zaghafte) Verzugszinspflicht ein: Der Versicherte hat Anspruch auf Verzugszinse, wenn sich die Auszahlung der Versicherungsleistung ohne sein Verschulden um mehr als zwei Jahre verzögert.

Wer sich vertieft mit dem neuen Gesetz auseinandersetzen möchte, konsultiert mit Gewinn die einschlägige Schwerpunktnummer der vom BSV herausgegebenen Zeitschrift «Soziale Sicherheit» $5 / 2002$ [1].

Wie gesagt: Der Schwerpunkt des ATSG liegt auf dem Verfahrensrecht. Immerhin ist die eine oder andere Änderung auch für die Ärzteschaft von praktischem Interesse. Konkret:

\section{Inkasso von Honorarforderungen: Zankapfel Abtretungsverbot}

Die Ausgangslage ist altbekannt: Praktisch alle Krankenkassen bestimmen in ihren Statuten oder allgemeinen Geschäftsbedingungen, dass der Versicherte seinen Rückerstattungsanspruch nicht abtreten darf, und zwar auch nicht seinem Arzt. In einigen Kantonen gibt es zwar mehr oder weniger explizite Regelungen, teilweise Gentlemen-Agreements, die Ausnahmen zulassen. In der Praxis ist das Ganze aber häufig mühsam durchzusetzen und daher mit viel Aufwand und Ärger verbunden. 
2 So auch Duc J-L. De la cession du droit aux prestations dans l'assurance-maladie obligatoire. SZS/RSAS 2002; $46: 276$.

Bei Bedarf finden Sie einen Mustertext unter www.fmh.ch $\rightarrow$ Recht $\rightarrow$ Mustervorlagen.
Dieser Punkt wird im neuen ATSG nun klargestellt: Zwar dehnt das Gesetz das bisher nur in der AHV geregelte Verbot der Abtretung von Versicherungsleistungen auf sämtliche Sozialversicherungszweige aus (Art. 22 ATSG). Für die soziale Krankenversicherung wird aber in Art. 42 Abs. 1 KVG eine explizite Ausnahme gemacht. Im Wortlaut: «In Abweichung von Art. 22 Absatz 1 ATSG kann dieser Anspruch dem Leistungserbringer abgetreten werden.»

Das Parlament hat diese Entscheidung bewusst getroffen: Das Abtretungsverbot «würde bedeuten, dass der Patient dem Arzt die Rückforderung an die Krankenkasse nicht mehr abtreten könnte. Deshalb wird für die Leistungserbringer generell in Art. 42 Absatz 1 KVG eine Ausnahme vom Abtretungsverbot vorgesehen» (Bundesblatt 1999, Nr. 23, S. 4572). Hintergrund dieser Regelung ist, die kontinuierliche medizinische Betreuung von Patienten mit finanziellen, sozialen oder medizinischen Problemen (z.B. Sucht) sicherzustellen. Überdies ist sie in Zeiten der sinkenden Zahlungsmoral ein Stück weit, wenn auch angesichts des Einfallsreichtums gewisser Patienten nur beschränkt, zur Absicherung von Honorarforderungen gegen notorisch säumige Schuldner geeignet.
Für die Krankenversicherer bedeutet die neue gesetzliche Regelung, dass sie ihre Statuten bzw. allgemeinen Geschäftsbedingungen ändern und im Bereich der gesetzlichen Grundversicherung künftig Abtretungserklärungen akzeptieren müssen [2]. Die Gefahr, dass Ärzte das neue Instrument missbrauchen könnten und somit das System des Tiers garant «unterhöhlen» würden, wie auch schon zu hören war, besteht mit Sicherheit nicht: Das Prozedere der Abtretung ist nicht nur mit einigem administrativen Aufwand verbunden (Ausfertigung und Versand der schriftlichen Erklärung*, Änderung der Stammdatei usw.); ein Arzt kann es sich auch nicht leisten, wahllos und unbedacht beispielsweise unbekannte Patienten zu brüskieren, und wird die Abtretung im eigenen Interesse nur in klaren und sinnvollen Fällen einsetzen.

Offen ist die Frage, ob die Patienten die gesamte Versicherungsleistung, ohne Rücksicht auf Selbstbehalt und Franchise, abtreten können, oder ob sich der Arzt mit der «Netto-Leistung» begnügen muss. Im zweiten Fall wäre dann, wenn der abtretungswillige Versicherte eine höhere Franchise vereinbart hat, der vom Parlament anvisierte Zweck der Sicherstellung der Behandlung faktisch zum Scheitern verurteilt. Sollten die Krankenkassen in diesem Punkt nicht zu einer vernünftigen Lösung Hand bieten, werden wohl die Gerichte das letzte Wort haben. 